\title{
A selective inhibitor of the immunoproteasome subunit LMP7 blocks cytokine production and attenuates progression of experimental arthritis
}

\author{
Tony Muchamuel ${ }^{1,4}$, Michael Basler ${ }^{2-4}$, Monette A Aujay ${ }^{1}$, Erika Suzuki ${ }^{1}$, Khalid W Kalim ${ }^{3}$, Christoph Lauer ${ }^{3}$, \\ Catherine Sylvain ${ }^{1}$, Eileen R Ring ${ }^{1}$, Jamie Shields ${ }^{1}$, Jing Jiang ${ }^{1}$, Peter Shwonek ${ }^{1}$, Francesco Parlati ${ }^{1}$, \\ Susan D Demo ${ }^{1}$, Mark K Bennett ${ }^{1}$, Christopher J Kirk ${ }^{1} \&$ Marcus Groettrup ${ }^{2,3}$ \\ The immunoproteasome, a distinct class of proteasome found predominantly in monocytes and lymphocytes, is known to \\ shape the antigenic repertoire presented on class I major histocompatibility complexes (MHC-I). However, a specific role \\ for the immunoproteasome in regulating other facets of immune responses has not been established. We describe here the \\ characterization of PR-957, a selective inhibitor of low-molecular mass polypeptide-7 (LMP7, encoded by Psmb8), the \\ chymotrypsin-like subunit of the immunoproteasome. PR-957 blocked presentation of LMP7-specific, MHC-I-restricted antigens \\ in vitro and in vivo. Selective inhibition of LMP7 by PR-957 blocked production of interleukin-23 (IL-23) by activated monocytes \\ and interferon- $\gamma$ and IL-2 by T cells. In mouse models of rheumatoid arthritis, PR-957 treatment reversed signs of disease \\ and resulted in reductions in cellular infiltration, cytokine production and autoantibody levels. These studies reveal a unique \\ role for LMP7 in controlling pathogenic immune responses and provide a therapeutic rationale for targeting LMP7 in \\ autoimmune disorders.
}

Multiple aspects of inflammatory responses, including cytokine production and antigen processing for presentation on MHC-I are regulated by the ubiquitin-proteasome system ${ }^{1,2}$. In most cells, the $26 \mathrm{~S}$ (or constitutive) proteasome contains the catalytic subunits $\beta 5, \beta 1$ and $\beta 2$, accounting for chymotrypsin-like, caspase-like and trypsinlike activities, respectively ${ }^{3}$. However, in cells of hematopoietic origin, particularly lymphocytes and monocytes, the proteasome catalytic subunits are encoded by homologous genes that code for the LMP7, LMP2 and multicatalytic endopeptidase complex subunit-1 (MECL-1) proteins $s^{4}$. These immunoproteasome subunits can also be induced in nonhematopoietic cells after exposure to inflammatory cytokines. Studies in knockout mice have demonstrated a role for immunoproteasome subunits in generating MHC-I ligands, establishing the naive $\mathrm{CD}^{+} \mathrm{T}$ cell repertoire and shaping cytotoxic $\mathrm{T}$ cell responses ${ }^{6-8}$. However, the contribution of the immunoproteasome to other aspects of immune cell function, such as the regulation of cytokine production, has not been described.

Small molecule inhibitors are useful tools for probing the role of the proteasome in immune responses ${ }^{9}$. Bortezomib, a dipeptide boronate approved for the treatment of multiple myeloma ${ }^{10}$, inhibits cytokine production in vitro and inflammation in vivo ${ }^{11,12}$. However, most well characterized proteasome inhibitors mediate equivalent inhibition of both proteasome chymotrypsin-like activities ( $\beta 5$ and LMP7) ${ }^{13-15}$ and have considerable toxicities that probably limit their clinical utility in chronic inflammatory diseases such as rheumatoid arthritis ${ }^{16}$. We describe here the immunomodulatory activity of an LMP7-selective peptide-ketoepoxide proteasome inhibitor related to carfilzomib ${ }^{15}$, a $\beta 5$ and LMP7-targeted molecule currently in clinical development for the treatment of multiple myeloma.

\section{RESULTS}

\section{PR-957 selectively inhibits LMP7}

We generated PR-957 (Fig. 1a) during a medicinal chemistry effort focused on the discovery of inhibitors with selectivity for immunoproteasome subunits. Like carfilzomib, PR-957 contains a ketoepoxide pharmacophore that covalently modifies proteasomal N-terminal threonine active sites ${ }^{15}$. We first determined the proteasome active site selectivity of PR-957 with a subunit-specific ELISA to quantify occupied proteasome active sites in intact cells ${ }^{17}$. In MOLT- 4 cells (a human leukemia cell line), which express both forms of the proteasome, PR-957 was 20- to 40-fold more selective for LMP7 over the next most sensitive sites, $\beta 5$ or LMP2 (Fig. 1b). In human peripheral blood mononuclear cells (PBMCs), in which constitutive proteasome subunit abundance was at the lower limit of detection (data not shown), PR-957 at concentrations $<100 \mathrm{nM}$ inhibited LMP7 activity by $>80 \%$ with minimal inhibition of LMP2 or MECL-1 (Fig. 1c).

${ }^{1}$ Proteolix, Inc., South San Francisco, California, USA. ${ }^{2}$ Biotechnology Institute Thurgau at Constance University, Kreuzlingen, Switzerland. ${ }^{3}$ Division of Immunology, Department of Biology, University of Constance, Konstanz, Germany. ${ }^{4}$ These authors contributed equally to this work. Correspondence should be addressed to C.J.K. (ckirk@proteolix.com) or M.G. (Marcus.Groettrup@uni-konstanz.de). 
Figure 1 PR-957 selectively targets LMP-7 activity in cells and tissues. (a) Structure of PR-957. (b) Active-site ELISA analysis of MOLT-4 (human T cell leukemia) cells exposed to PR-957 at varying concentrations $(1 \mathrm{nM}-10 \mu \mathrm{M})$ for $1 \mathrm{~h}$. Activities of the LMP7, LMP2, and MECL-1 subunits of the immunoproteasome and the $\beta 5$, $\beta 1$ and $\beta 2$ subunits of the constitutive proteasome were normalized to values derived from DMSO-treated controls. (c) Activity of LMP7, LMP2 and MECL-1 in human PBMCs incubated with the indicated concentrations of PR-957. Data are presented as the mean relative activity from three separate donors versus DMSO controls. (d) Quantification of hydrolysis of fluorogenic substrates for chymotrypsin-like (LLVY-AMC) and trypsin-like (Val-Gly-Arg-AMC VGR-AMC) activity of $20 \mathrm{~S}$ proteasomes from livers of LCMV-WE-infected WT and Psmb8 ${ }^{-1-}$ mice at various inhibitor concentrations. Data are presented as the means \pm s.d. relative activity from triplicate assays. The experiments were repeated three times with similar results. (e) Flow cytometry analysis of $\mathrm{H}-2 \mathrm{D}^{\mathrm{b}}$ and $\mathrm{H}-2 \mathrm{~K}^{\mathrm{b}}$ surface expression on splenocytes derived from WT or $\begin{array}{ll}\text { expression on splenocytes derived from WT or } & \text { [PR-957] (nM) } \\ \text { Psmb8 }^{-1-} \text { mice treated with the indicated } & \end{array}$
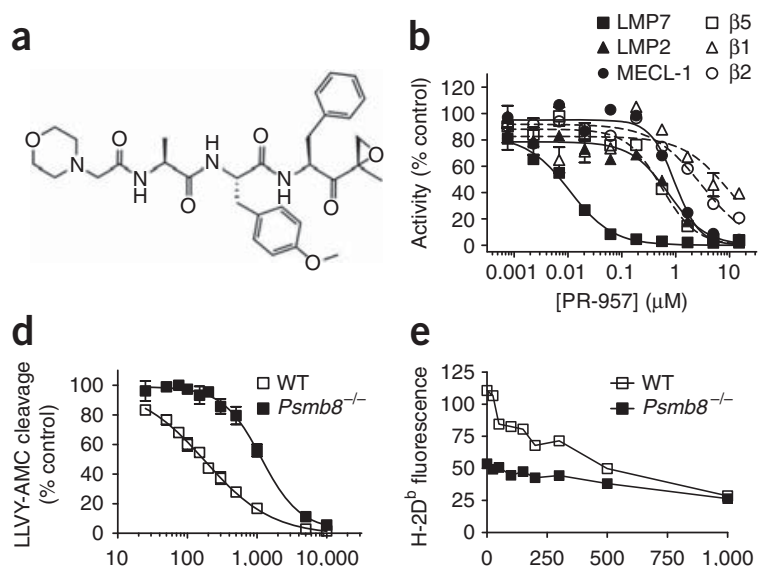

\section{e}

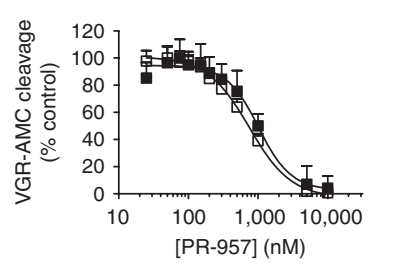

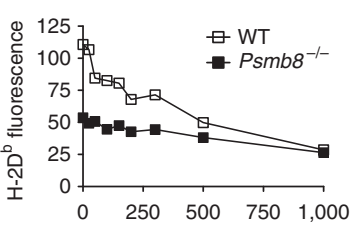

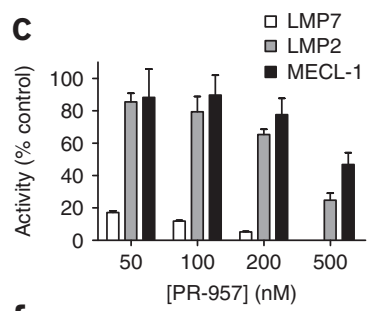

f
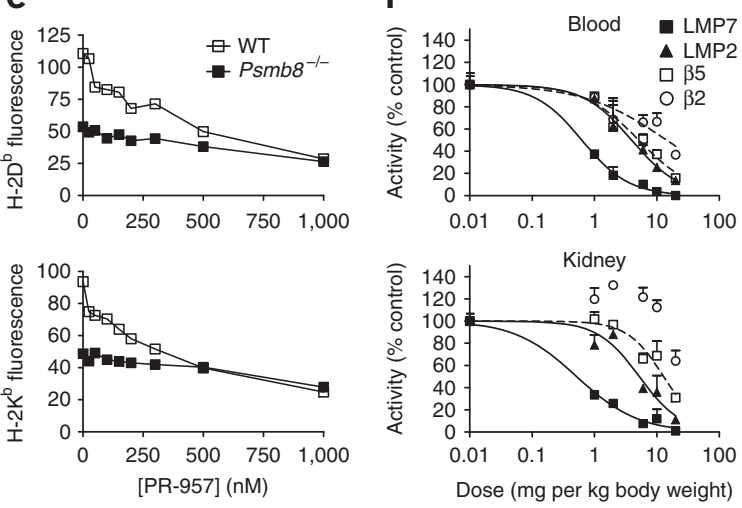
concentration of PR-957. (f) Active-site ELISA analysis of the activity of LMP7, LMP2, $\beta 5$ and $\beta 2$ in whole-blood and kidney samples (taken $1 \mathrm{~h}$ after dosing) from BALB/c mice given an i.v. administration of PR-957 at doses ranging from 1 to 20 mg per kg body weight. Data were normalized to the average activity of vehicle-treated mice and are presented as the average relative activity \pm s.e.m. $(n=3)$.

Higher concentrations resulted in substantial inhibition of LMP2 and MECL-1 (Fig. 1c). Selective inhibition of LMP7 did not perturb overall proteasome function, as evidenced by a lack of polyubiquitinylated protein accumulation or stabilization of p53 in MOLT-4 cells or accumulation of a destabilized GFP reporter in 293 cells (Supplementary Fig. 1). Selectivity for LMP7 was lower in A20 mouse lymphoma cells than in human cells (Supplementary Fig. 2a), possibly reflecting cross-species sequence differences in LMP7 (ref. 18).

We also evaluated PR-957 activity on purified proteasomes derived from the livers of lymphocytic choriomeningitis virus (LCMV)-WEinfected wild-type (WT) or LMP7-deficient $\left(P s m b 8^{-I-}\right)$ mice, which show reduced proteasomal incorporation of LMP2 and MECL-1 owing to a lack of LMP7 (refs. 19,20). LCMV infection results in replacement of the constitutive proteasome active site subunits in hepatocytes with their immunoproteasome counterparts, with LMP7 mediating chymotrypsin-like activity ${ }^{21,22}$. At $25-300$ nM, PR-957 inhibited chymotrypsin-like activity of liver proteasomes from WT but not $P s m b 8^{-1-}$ mice (Fig. 1d). Trypsin-like activity, in contrast, was unaffected by PR-957 over the same concentration range and was independent of genotype at higher concentrations (Fig. 1d). In assays of purified human proteasome preparations, PR-957 acted as a timedependent inhibitor (indicative of a covalent reaction expected for the ketoepoxide pharmacophore ${ }^{15}$ ) that was 10 - to 15 -fold more selective for the immunoproteasome (Supplementary Table 1). The lower selectivity seen with purified proteasomes may have arisen from cleavage of the substrate, Leu-Leu-Val-Tyr-7-amino-4-methylcoumarin (LLVY-AMC), by LMP2 (ref. 23).

As splenocytes derived from LMP7-deficient mice show a reduction in MHC-I surface expression on lymphocytes ${ }^{19}$, we compared the effect of PR-957 on MHC-I H-2 ${ }^{\mathrm{b}}$ and MHC-I H-2K $\mathrm{K}^{\mathrm{b}}$ expression in WT and $P s m b 8^{-1-}$ mouse splenocytes. Expression of MHC-I in WT mice was reduced up to $50 \%$ at LMP7-selective concentrations of
PR-957 ( $\leq 300 \mathrm{nM}$ ) (Fig. 1e). In contrast, basal MHC-I expression in Psmb8 ${ }^{-1-}$ splenocytes, which is $\sim 50 \%$ lower relative to WT levels, was not further affected by PR-957 treatment (Fig. 1e). PR-825, a selective inhibitor of $\beta 5$, decreased MHC-1 expression on $P s m b 8^{-l-}$ splenocytes (Supplementary Fig. 2d). Because viability was not substantially affected by either PR-957 or PR-825 (data not shown), the reduced MHC-I expression was due to reduced peptide supply, similar to observations with $P s m b 8^{-1-}$ mice ${ }^{19}$.

We used the active-site ELISA to monitor proteasome subunit inhibition in blood and tissues (kidney, heart and spleen) in mice after intravenous (i.v.) administration of PR-957 at doses ranging from 1 to $20 \mathrm{mg}$ per $\mathrm{kg}$ body weight. Selective inhibition of LMP7 occurred at doses ranging from 1 to $10 \mathrm{mg}$ per kg body weight in both blood and kidney (Fig. 1f). The dose response for LMP7 inhibition in the kidney was comparable to that in blood (half-maximal inhibitory concentration $<1 \mathrm{mg}$ per kg body weight), indicating efficient tissue penetration. We noted similar inhibition profiles in heart and spleen (data not shown). We determined the maximum tolerated dose (MTD) of PR-957 in mice to be $30 \mathrm{mg}$ per $\mathrm{kg}$ body weight (Supplementary Table 2), indicating that inhibition of LMP7 is well tolerated. Taken together, these data demonstrate the selectivity of PR-957 for LMP7 in vitro and in vivo.

\section{PR-957 inhibits LMP7-specific antigen presentation}

The immunoproteasome shapes presentation of both endogenous and virally derived MHC-I-restricted antigens. We first investigated the effect of PR-957 on the endogenously expressed, LMP7-dependent epitope $\mathrm{UTY}_{246-254}$, derived from the male minor antigen $\mathrm{HY}^{24}$. At 300 nM PR-957, an LMP7-selective concentration (Fig. 1d and Supplementary Fig. 2a), presentation of $\mathrm{UTY}_{246-254}$ was decreased to a level near that of splenocytes derived from either female or $P$ smb8 ${ }^{-1-}$ male mice (Fig. 2a). To assess the effect of PR-957 on antigen presentation in vivo, we exposed splenocytes harvested from 
Figure 2 PR-957 blocks MHC-I-restricted presentation of LMP7-dependent epitopes. (a) Presentation of UTY $246-254$ by MHC-I by as analyzed by exposure to $\mathrm{H}-2 \mathrm{D}^{\mathrm{b}}-\mathrm{UTY} \mathrm{Y}_{246-254^{-}}$ specific, LacZ-expressing T cell hybridomas. Data are presented as the mean absorbance \pm s.d. of three replicate cultures. (b) Presentation of $\mathrm{UTY}_{246-254}$ by splenocytes after one $(1 \times)$ or two $(2 \times)$ daily i.v. administrations of $6 \mathrm{mg}$ per $\mathrm{kg}$ body weight PR-957. Data are presented as the mean absorbance \pm s.d. of three replicate cultures. (c) Presentation of viral epitopes in female C57BL/6 mice treated daily for $5 \mathrm{~d}$ with $6 \mathrm{mg}$ per kg body weight i.v. PR-957, as analyzed by flow cytometry for antigen-specific expression of IFN- $\gamma$. Data are presented as the percentage of $\mathrm{CD}^{+}$cells expressing IFN- $\gamma$. $P$ values are from unpaired $t$ test (MannWhitney). n.i., noninfected splenocytes. (d) Presentation of LCMV-specific epitopes by splenocytes treated with 300 nM PR-957 and infected with LCMV-WE, as measured with peptide-specific T cell lines. For NP118 reactivity, BALB/c splenocytes were incubated with differing concentrations of PR-957. Antigenspecific CTL activation was assessed as described in c. Data are presented as the percentage of $\mathrm{CD}^{+}$cells expressing IFN- $\gamma$. (e) CTL activation in vivo, as measured in splenocytes from mice left untreated (NT) or treated with PR-957 for $5 \mathrm{~d}$. Mice were infected with LCMV-WE beginning $1 \mathrm{~d}$ after treatment initiation, and splenocytes were harvested and analyzed for IFN- $\gamma$ expression as described in $\mathbf{d} 8 \mathrm{~d}$ after infection. Data are presented as the percentage of CD8 ${ }^{+}$cells expressing IFN- $\gamma$. $P$ values are from unpaired $t$ test (Mann-Whitney). (f) Blood glucose concentrations, as measured in RIP-GP mice after $10 \mathrm{~d}$ of treatment with 6 mg per kg body weight PR-957 and infection with LCMV-WE (day 2 of treatment). Blood glucose concentrations were measured beginning $1 \mathrm{~d}$ after infection in two mice per time point. Data are presented as the mean blood glucose levels \pm s.d.
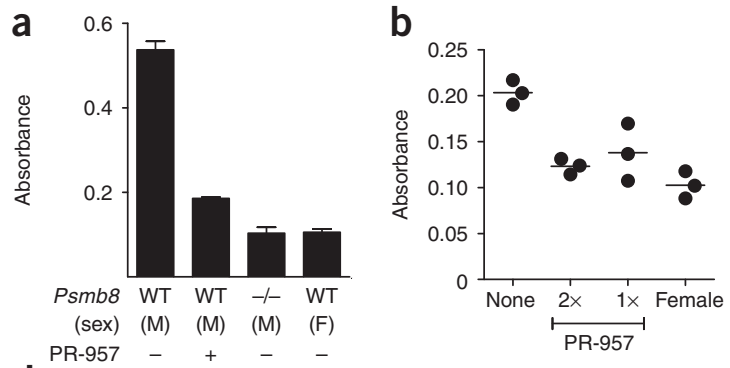

d
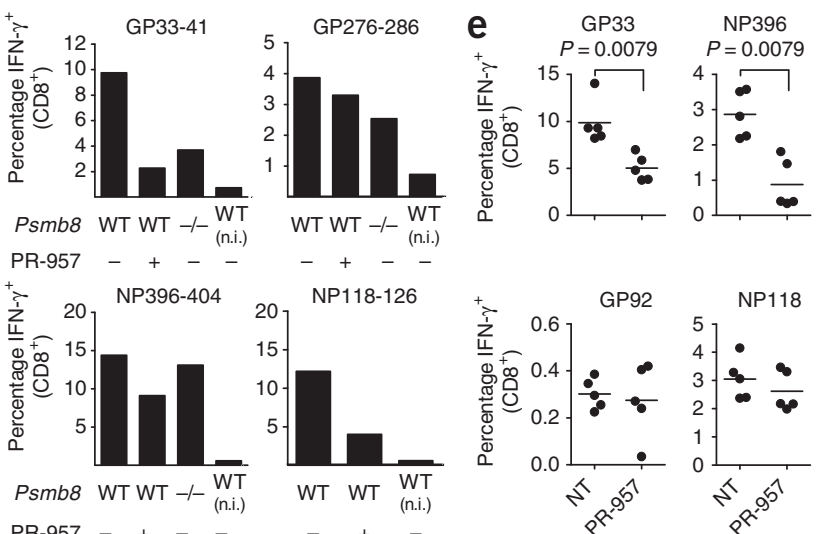
splenocytes after exposure to 300 nM PR-957,
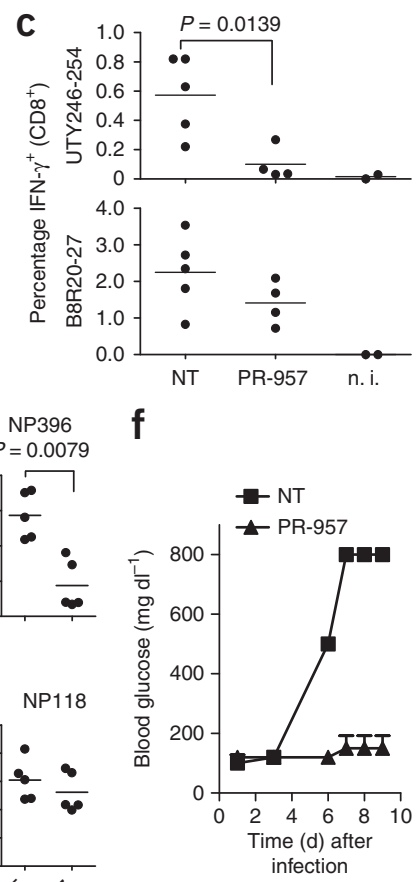

mice $1 \mathrm{~h}$ after a single or the second of two daily doses of PR-957 to $\mathrm{UTY}_{246-254}$-specific T cell hybridomas. A single administration was

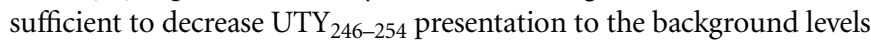
shown by splenocytes from female mice (Fig. $2 \mathbf{b}$ ). These data indicate that presentation of an endogenously expressed, LMP7-dependent epitope can be blocked by exposure to PR-957 in vitro or in vivo.

To assess the ability of PR-957 to affect the cytotoxic T lymphocyte response to virally encoded epitopes, we treated female mice with PR-957 for $5 \mathrm{~d}$ and infected them with recombinant vaccinia virus expressing the UTY protein (rVV-UTY) on day 2 of treatment. We immunized the mice $9 \mathrm{~d}$ before PR-957 treatment with peptide-loaded male-derived splenocytes to increase the frequency of UTY $246-254$ specific $\mathrm{T}$ cell precursors. We measured the $\mathrm{UTY}_{246-254}$-specific T cell response $8 \mathrm{~d}$ after infection by intracellular cytokine staining (ICS) for interferon- $\gamma$ (IFN- $\gamma$; Fig. 2c). PR-957 treatment suppressed the $\mathrm{UTY}_{246-254}$-specific response to levels equivalent to those in uninfected mice, whereas the response to the dominant vaccinia virusspecific epitope $\mathrm{B} \mathrm{R}_{20-27}$ was only slightly reduced (Fig. 2c). Viral titers on day 8 were tenfold higher in PR-957-treated mice as compared to untreated control mice, excluding a direct effect of PR-957 on viral replication (data not shown). We reasoned that the reduced viral clearance could be the result of the decreased UTY $246-254$ $\mathrm{T}$ cell response. Indeed, PR-957-treated mice infected with WT vaccinia virus (VV-WR) had similar viral titers as compared to untreated mice (data not shown), ruling out an effect of PR-957 on viral replication. Furthermore, PR-957 treatment for $5 \mathrm{~d}$ before VV-WR infection did not affect the number of natural killer cells $\left(\mathrm{NK} 1.1^{+}\right)$, dendritic cells $\left(\mathrm{CD} 11 \mathrm{c}^{+}\right), \mathrm{B}$ cells $\left(\mathrm{CD} 19^{+}\right), \mathrm{CD}^{+} \mathrm{T}$ cells, $\mathrm{CD}^{+} \mathrm{T}$ cells or macrophages $\left(\mathrm{F} 4 / 80^{+}\right)$as compared to numbers in untreated mice (data not shown). These data suggest that PR-957-treated mice mount a normal vaccinia virus-specific $T$ cell response but have an impaired response to the LMP7-specific epitope $\mathrm{UTY}_{246-254}$.

To evaluate the effect of PR-957 on the presentation of LCMVderived epitopes, we infected splenocytes with LCMV in vitro before treatment. We assessed the presentation of the MHC-I-restricted LCMV epitopes glycoprotein 33-41 (GP33), glycoprotein 276-286 (GP276), nucleoprotein 396-404 (NP396) and nucleoprotein 118-126 (NP118) using peptide-specific cytotoxic T lymphocyte (CTL) lines in ICS assays for IFN- $\gamma$ (Fig. 2d). PR-957 markedly decreased presentation of GP33 and NP118 while having a minimal effect on GP276 and NP396 presentation (Fig. 2d). The dependence of GP33 on LMP7 confirms the results from an earlier study using in vitro-infected macrophages from $P s m b 8^{-1-}$ mice ${ }^{6}$.

We assayed splenocytes from LCMV-infected and PR-957-treated mice $8 \mathrm{~d}$ after infection for responses to LCMV epitopes. Compared to untreated mice, PR-957-treated mice had CTL responses to GP33 and NP396 that were reduced by $50 \%$ and $70 \%$, respectively $(P<0.01)$, whereas responses to GP92 and GP118 were not markedly altered (Fig. 2e). The CTL response in BALB/c mice, which is dominated by NP118-specific CTLs, was lowered with PR-957 treatment by approximately one third (data not shown). We did not observe any differences in CTL response in LMP7-deficient mice treated with PR-957 compared to untreated gene-deficient mice (data not shown). Virus titers $4 \mathrm{~d}$ after infection and the numbers of various cell populations characterized by the surface markers CD8, CD4, NK1.1, CD11c, F4/80 and CD19 on day 8 after infection were not altered in PR-957-treated mice as compared to untreated mice (data not shown). The more 
Figure 3 PR-957 inhibits production of inflammatory cytokines in human PBMCs. (a) Proteasome subunit activity of $200 \mathrm{nM}$ PR-957 or 125 nM PR-825 were derived from dose response curves shown in Figure $\mathbf{1 b}$ and Supplementary Figure 2c. (b) Cytokine production by PBMCs from normal healthy donors ( $n=4-6$ donors per cytokine) after treatment with either 200 nM PR-957 or 125 nM PR-825 for $1 \mathrm{~h}$ and exposure to $1 \mathrm{ng} \mathrm{ml} \mathrm{m}^{-1}$ LPS for $24 \mathrm{~h}$. Supernatants were analyzed for expression of IL-23, TNF- $\alpha$ and IL- 6 by ELISA. Values are presented as the mean relative level \pm s.e.m. as compared to DMSO-treated controls. ${ }^{*} P<0.05$ and ${ }^{*} P<0.01$ versus DMSO controls by paired $t$ test of absolute cytokine levels. (c) T cell activation in PBMCs, as measured after exposure to PR-957 at the indicated concentrations for $1 \mathrm{~h}$ and stimulation with plate-bound antibodies specific for CD3 and CD28. Supernatants were analyzed for levels of IFN- $\gamma$ and IL- 2 by ELISA.

a
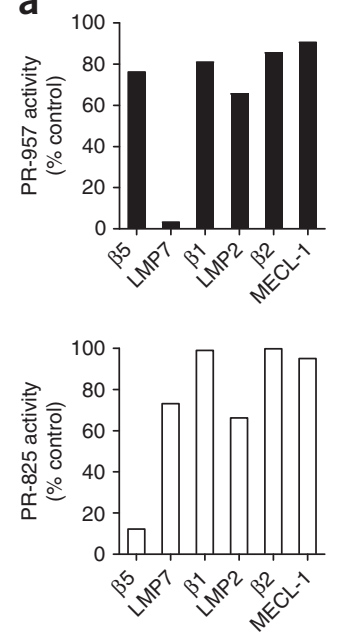

b

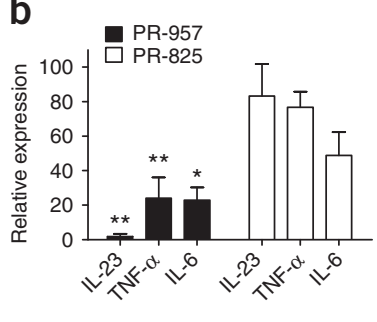

C

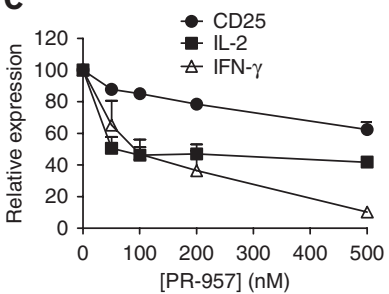

d

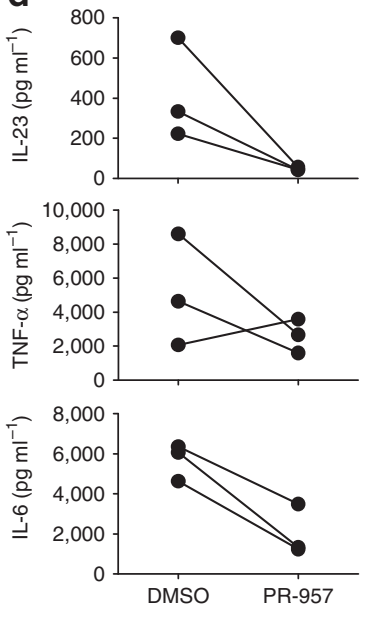

The cells were also analyzed for cell surface expression of CD25 (on CD4+ cells). Values are presented as the mean relative level as compared to DMSOtreated controls. (d) PBMCs from individuals with rheumatoid arthritis were exposed to DMSO or $200 \mathrm{nM}$ PR-957 and stimulated as described in b. Supernatants were analyzed for IL-23, IL-6 and TNF- $\alpha$ by ELISA.

prominent reduction in the CTL response in PR-957-treated mice as compared to $P s m b 8^{-1-}$ mice ${ }^{6,25}$ may reflect compensatory incorporation of $\beta 5$ into immunoproteasomes in $P s m b 8^{-1-}$ mice.

Transgenic mice expressing the LCMV glycoprotein exclusively in pancreatic $\beta$-islet cells via the rat insulin promoter (RIP-GP mice) develop diabetes after infection with LCMV as a result of islet cell destruction by glycoprotein-specific CTLs ${ }^{26}$. To assess the effect of PR-957 in this model, we directed the $\mathrm{T}$ cell response in these mice toward GP33 by adoptive transfer of Thy $1.2^{+}$cells derived from $\mathrm{H}-2 \mathrm{D}^{\mathrm{b}}$-GP33-specific $\mathrm{T}$ cell receptor-transgenic mice before LCMV infection ${ }^{27}$. We verified that the LCMV-specific T cell response was predominantly directed against GP33 by ICS (data not shown) and monitored islet cell destruction by the increase in blood glucose levels. PR-957 treatment $2 \mathrm{~d}$ before infection completely protected mice from signs of diabetes (Fig. 2f). Together, these data demonstrate that PR-957 treatment alters $\mathrm{CD}^{+} \mathrm{T}$ cell responses in virally infected mice.

\section{Inhibition of LMP7 blocks cytokine production in vitro}

Cytokine production in endotoxin-stimulated monocytes is blocked by exposure to proteasome inhibitors that target both $\beta 5$ and LMP7 (ref. 11). To evaluate the impact of selective immunoproteasome inhibition, we compared the cytokine production in endotoxinstimulated PBMCs exposed to PR-957 or PR-825 at concentrations resulting in selective inhibition ( $>80 \%$ ) of LMP7 or $\beta 5$, respectively (Fig. 3a). LMP7 inhibition blocked production of IL-23 by $>90 \%$ and of tumor necrosis factor- $\alpha$ (TNF- $\alpha$ ) and IL-6 by $\sim 50 \%$ (Fig. $3 \mathbf{b}$ ). Higher concentrations of PR-957, which induce inhibition of LMP2 and MECL-1, further decreased secretion of TNF- $\alpha$ and IL-6, suggesting that these subunits have a role in cytokine regulation (data not shown). Selective inhibition of $\beta 5$, in contrast, did not substantially affect cytokine release (Fig. 3b). Neither PR-957 nor PR-825 inhibited nuclear factor- $\kappa \mathrm{B}$ activity in a reporter cell line at selective concentrations (Supplementary Fig. 3), suggesting that LMP7 regulates cytokine production via nuclear factor- $\mathrm{\kappa B}$-independent pathways. These
Figure 4 PR-957 blocks cytokine production by mouse splenocytes and blocks $T$ cell differentiation. (a) Cytokine production by splenocytes from WT or Psmb8 ${ }^{-1-}$ mice treated with $300 \mathrm{nM}$ PR-957 for $1 \mathrm{~h}$ and then exposed to $5 \mu \mathrm{g} \mathrm{ml} \mathrm{m}^{-1}$ LPS for $24 \mathrm{~h}$. Supernatants were analyzed for IL-6 concentration by ELISA. (b) Cytokine production by splenocytes stimulated with plate-bound antibodies specific for CD3 and CD28 for $24 \mathrm{~h}$ and analyzed for IFN- $\gamma$ production by ELISA. Cytokine concentrations are presented as the mean values \pm s.e.m. from triplicate wells. (c) Differentiation of $\mathrm{CD} 4^{+} \mathrm{T}$ cells from WT mice exposed to the indicated concentrations of PR-957 and stimulated with antibody to CD3 and antibody to CD28 (CD3 + $\mathrm{CD} 28 \mathrm{Ab})$ in the presence of TGF- $\beta$ and IL- 6 and antibodies to IL- 4 and IFN- $\gamma$ was measured in 3-d cultures. IL-17 expression was detected by ICS. IL-17 expression in activated T cells cultured in the absence of $T_{H} 17$-polarizing conditions is shown as a comparison (no cytokines). The analysis was performed twice in triplicates, yielding similar results. Values reflect the percentage of $\mathrm{CD}^{+}$cells that are also $\mathrm{IL}^{-17 \mathrm{~A}^{+}}$. b
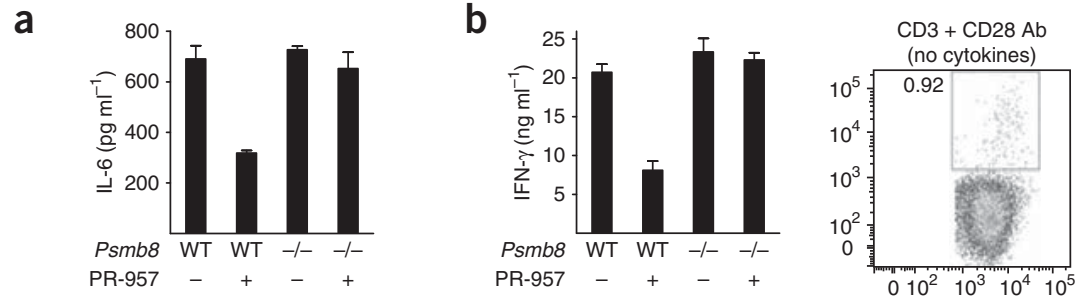

C

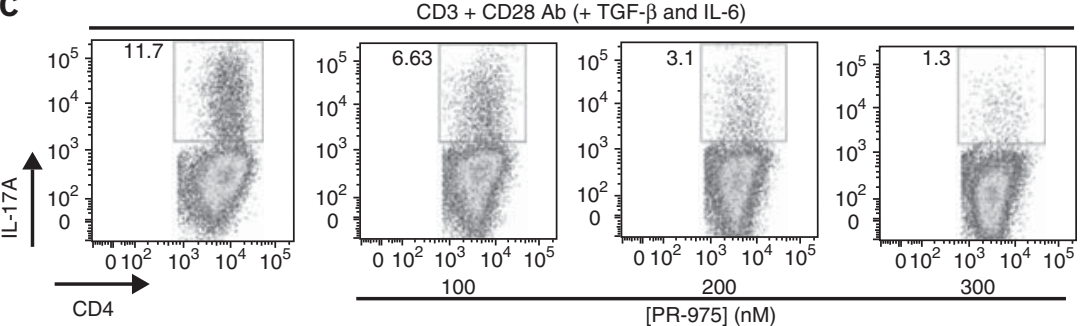


Figure 5 PR-957 administration reduces the severity of CAIA and blocks molecular and cellular joint inflammation. (a) Arthritis was induced in BALB/c mice with antibodies specific for type II collagen ( $m A b)$ and endotoxin. On day 4 , when disease was present in all animals, mice were randomized and treated with vehicle or PR-957 at 2, 6 or $10 \mathrm{mg}$ per $\mathrm{kg}$ body weight. Dosing was repeated on days 6 and 8 , and clinical scores (0-4 per paw; $n=7 /$ group) were followed until day 14. Data are presented as the mean clinical score \pm s.e.m. $P$ values are from a two-way ANOVA followed by Bonferroni post hoc comparison. (b) Joint expression of mRNA for the indicated proteins, as measured in mice with CAIA induced as described in a. Mice received either vehicle or $10 \mathrm{mg}$ per $\mathrm{kg}$ body weight PR-957 on days 4 and 6 after collagen-specific antibody challenge. Paws were collected on day 7 and analyzed by quantitative RT-PCR ( $\beta$-actin-normalized) for expression. Data are mean normalized values \pm s.e.m. ( $n=5$ /group), and $P$ values are derived from an unpaired $t$ test (Mann-Whitney). ICAM-1, intercellular adhesion molecule-1; iNOS, inducible nitric oxide synthase; Cox-2, cyclooxygenase-2. (c) CAIA was induced in BALB/c mice as described in a. On day 4 , mice with active disease were randomized to receive vehicle, $10 \mathrm{mg}$ per $\mathrm{kg}$ body weight PR-957 (i.v.) or $10 \mathrm{mg}$ per kg body weight etanercept (subcutaneously) on days 4, 6 and 8 . Data are represented as means \pm s.e.m. ( $n=7 /$ group). P values are from a two-way ANOVA followed by Bonferroni post hoc comparison. (d) Histological assessment of tarsal joints on day 14 in mice with CAIA (as described in a) and treated with either vehicle or PR-957 (10 mg per kg body weight) on days 4, 6 and 8 . Scale bar, $100 \mu \mathrm{m}$. (e) Histological scores (day 14 ) from individual PR-957-treated and etanercept-treated mice ( $n=5 / g r o u p)$ are shown. ${ }^{* *} P<0.01$ and ${ }^{* * *} P<0.001$ versus vehicle. $P$ values are from a one-way ANOVA followed by Bonferroni post hoc comparison. data indicate that LMP7 selectively regulates inflammatory cytokine production in endotoxin-stimulated PBMCs.

To determine the effect of PR-957 on $\mathrm{T}$ cell activation, we stimulated PR-957-treated PBMCs with antibodies to CD3 and CD28. IFN- $\gamma$ release was inhibited by $\sim 60 \%$ at LMP7-selective concentrations of PR-957 and by $\sim 90 \%$ at higher concentrations (Fig. 3c). Production of IL-2 was inhibited by $\sim 50 \%$ at all tested concentrations (Fig. 3c), indicating that both LMP7-dependent and immunoproteasome-independent pathways contribute to the expression of this cytokine. Similar to our findings with endotoxin stimulation, PR-825 treatment (at $\beta 5$-selective concentrations) did not affect production of IFN- $\gamma$ or IL-2 (data not shown). Early T cell activation, as measured by CD25 expression, was largely unaffected by PR-957, similar to previous findings in $P s m b 8^{-1-}$ mice ${ }^{28}$.

We also determined the effect of PR-957 on cells derived from individuals with active rheumatoid arthritis. Similar to the findings described above, selective inhibition of LMP7 blocked IL-23 production by $\sim 80 \%$ and IL-6 and TNF secretion by $\sim 50 \%$ (Fig. 3d). Taken together, these data suggest that LMP7 regulates inflammatory cytokine production in cells from both normal healthy individuals and those with rheumatoid arthritis.

We compared the effect of PR-957 on cytokine release in WT and Psmb8-deficient mouse splenocytes. PR-957 blocked production of endotoxin-stimulated IL-6 (Fig. 4a) and T cell-mediated IFN- $\gamma$ production (Fig. 4b) in WT but not $P s m b 8^{-1-}$ splenocytes. The lack of effect in $P s m b 8^{-/-}$cells indicates that PR-957 affects cytokine production through specific inhibition of LMP7. The fact that $P s m b 8^{-I-}$ and WT splenocytes did not differ in IL-6 or IFN- $\gamma$ production may be the result of compensatory incorporation of $\beta 5$ in proteasomes from $\mathrm{Psmb8}^{-1-}$ cells.

We next tested the effect of LMP7 on T helper type $17\left(\mathrm{~T}_{\mathrm{H}} 17\right)$ differentiation. We exposed $\mathrm{CD}^{+}{ }^{+} \mathrm{T}$ cells from WT mice to PR-957 during stimulation and differentiation into $\mathrm{T}_{\mathrm{H}} 17$ cells through the addition of IL- 6 and transforming growth factor- $\beta$ (TGF- $\beta$ ). Inhibition of LMP7 prevented differentiation of $\mathrm{T}_{\mathrm{H}} 17$ cells, as measured by IL-17 expression after $3 \mathrm{~d}$ of cell culture (Fig. 4c). These data suggest that LMP7 controls both the early activation of T cells as well as differentiation into inflammatory effector cells in the presence of polarizing cytokines.

\section{LMP7 inhibition ameliorates disease in mouse arthritis}

To determine the impact of immunoproteasome inhibition on inflammatory responses in vivo, we evaluated PR-957 in two different mouse models of rheumatoid arthritis: collagen antibody-induced arthritis (CAIA) and collagen-induced arthritis (CIA). In the CAIA model, we randomized mice challenged with antibodies to collagen and endotoxin to treatment groups after the onset of clinical symptoms. PR-957 blocked disease progression in a dose-dependent manner and completely ameliorated visible signs of disease at the highest dose (Fig. 5a). Inhibition of LMP7 alone was sufficient to block disease progression, as evidenced by the therapeutic response to PR-957 administered at $2 \mathrm{mg}$ per kg body weight (Fig. 1f), that is, less than one-tenth the MTD. Carfilzomib inhibited disease progression by $\sim 50 \%$ but only at the MTD, whereas PR-825, at a dose resulting in $>80 \%$ inhibition of $\beta 5$ (data not shown), had no effect in this model (Supplementary Fig. 4b). Anti-inflammatory responses induced by PR-957 were rapid and long lasting, with disease regression evident $24 \mathrm{~h}$ after the first dose (Fig. 5), whereas a single dose, administered either intravenously or subcutaneously, resulted in complete amelioration of disease (Supplementary Fig. 4a). RT-PCR analysis of tarsal joints on day 7 showed that PR-957 treatment lowered expression of multiple inflammatory mediators, including TNF- $\alpha$ and IL-6 (Fig. 5b). When compared to anti-TNF- $\alpha$ therapy (etanercept), PR-957 mediated a more rapid resolution of clinical symptoms (Fig. 5c). We observed a statistically significant decrease in inflammatory infiltration and subsequent bone erosion in PR-957-treated mice (Fig. 5d,e). As 
Figure 6 PR-957 treatment reduces the severity of active CIA. (a) Arthritis was induced in DBA/1 J mice by immunization with type II collagen on day 0 . On day 25 , when disease symptoms were present (average score $=2$ ), mice were randomized to groups and treated with either vehicle, or PR-957 at 2, 6 and $10 \mathrm{mg}$ per $\mathrm{kg}$ body weight on days 25, 27, 29, 31 and 33 . Disease was scored as in Figure $\mathbf{5}$ and the data, presented as the mean clinical score \pm s.e.m. ( $n=10$ per group). $P$ values are from a two-way ANOVA followed by Bonferroni post hoc comparison. (b) Plasma levels of collagenspecific antibody titers were measured by ELISA on day 35 in mice treated with either vehicle or $10 \mathrm{mg}$ per kg body weight PR-957 as described in a. Data are from individual mice, and the $P$ value is from an unpaired $t$ test (Mann-Whitney). (c) Serum levels of COMP, as measured by ELISA on day 35 in mice treated as described in $\mathbf{b}$. Values presented are the mean units per $\mathrm{ml}$ \pm s.e.m., and the $P$ value is derived from an unpaired $t$ test. (d,e) Histological assessment of

tarsal joints from mice treated with vehicle or PR-957 (10 mg per kg body weight), as described in a, was carried out on day 35 . A representative tarsal joint from each treatment group (d) and histology scores of individual mice (e) are shown. Scale bar, $100 \mu \mathrm{m}$. The $P$ value is derived from an unpaired $t$ test ( $n=5$ per group). (f) CIA was induced as in a, and on day 25 mice with disease scores between 4 and 6 were randomized to groups receiving either vehicle (i.v.), $10 \mathrm{mg}$ per kg body weight PR-957 (i.v.) or $10 \mathrm{mg}$ per kg body weight etanercept (subcutaneously) on the dose schedule described in a. Data, presented as the mean clinical score \pm s.e.m. ( $n=10$ per group). ${ }^{* *} P<0.001$ versus vehicle. $P$ values are from a two-way ANOVA followed by Bonferroni post hoc comparison.

this model of rheumatoid arthritis is $\mathrm{T}$ cell independent ${ }^{29}$, the therapeutic effect of PR-957 was not due to changes in MHC-I antigen presentation.

PR-957 treatment also induced a rapid therapeutic response in the $\mathrm{T}$ and $\mathrm{B}$ cell-dependent CIA model (Fig. 6a). Immunoproteasome inhibition was associated with a decrease in circulating levels of autoantibodies (Fig. 6b) and collagen oligomeric matrix protein (COMP), a marker for cartilage breakdown (Fig. 6c). Lower COMP levels corresponded with inhibition of bone erosion and joint inflammation (Fig. 6d,e). In a model of aggressive disease, PR-957 was more effective than etanercept (Fig. 6f). As etanercept showed equivalent activity to PR-957 in mice with less severe disease (data not shown), we surmise that TNF- $\alpha$ inhibition alone is insufficient to promote long lasting therapeutic activity in mice with aggressive polyarthritis.

\section{DISCUSSION}

PR-957 is the first proteasome inhibitor described that is selective for the chymotrypsin-like subunit of the immunoproteasome, and it represents a powerful tool for understanding the role of LMP7 in immune responses. We demonstrated the selectivity of PR-957 for LMP7 by three different methods: an active-site ELISA to monitor subunit activity, biochemical assays using purified human proteasomes and proteasomes from WT and $P s m b 8^{-1-}$ mice, and monitoring of MHC-1 expression in WT and $P s m b 8^{-1-}$ splenocytes. Exposure to PR-957 in vitro and in vivo blocked presentation of LMP7-specific epitopes (UTY ${ }_{246-254}$ and GP33), highlighting the effect of selective inhibition of this subunit. PR-957 treatment allows for an assessment of LMP7 function in immune cells and mice with normal proteasome architecture. Compared to immunoproteosomes from wild-type mice, immunoproteasomes from $P s m b 8^{-l-}$ mice contain increased amounts of $\beta 5$ and decreased LMP2 and MECL-1, which may alter proteasome function within immune effector cells ${ }^{19,30-32}$. These compensatory changes may mask LMP7-specific functions in complex cellular processes such as inflammatory responses.
The data presented here show a unique role for immunoproteasome subunits in cytokine production and suggest that the effects of dual $\beta 5$-LMP7 inhibitors such as bortezomib reflect immunoproteasome inhibition $^{33,34}$. Inhibition of LMP7, but not $\beta 5$, blocked cytokine production in LPS-stimulated PBMCs. Our results extend findings demonstrating that proteasome subunits have distinct roles in regulating cellular protein turnover ${ }^{35}$ by showing that cytokine release depends on specific immunoproteasome subunits. A specific role for LMP7 in cytokine production may help explain a previous report of altered bacterial clearance despite normal CTL generation in $P s m b 8^{-1-}$ mice $^{36}$.

The therapeutic impact of LMP7 inhibition in mouse models of rheumatoid arthritis includes reductions in inflammation, cytokine gene expression and serum autoantibody levels. Because we observed similar findings in lymphocyte-dependent and lymphocyte-independent models of rheumatoid arthritis, and because LMP7 inhibition blocks both $\mathrm{T}$ cell and monocyte function in vitro, PR-957 probably modulates the activity of multiple effector cell types in these disease models. Furthermore, LMP7 inhibition prevents the production of cytokines driving $\mathrm{T}_{\mathrm{H}} 17$ generation and IL-17 production in $\mathrm{T}$ cells cultured in the presence of polarizing cytokines, suggesting that LMP7 regulates $\mathrm{T}$ cell function at multiple levels.

It is noteworthy that PR-957 induces an anti-inflammatory response at doses less than one tenth the MTD, in contrast to nonselective inhibitors, such as bortezomib ${ }^{11,12}$ and carfilzomib (Supplementary Fig. 4), which induce anti-inflammatory responses at the MTD. The increased therapeutic margin with an immunoproteasome selective inhibitor provides a rationale for clinical development of this agent in autoimmune disorders such as rheumatoid arthritis. The therapeutic response to PR-957 treatment was similar to entanercept for both clinical scores and histologic progression of disease in the CAIA model but was associated with a more rapid response, possibly owing to direct effects on multiple cytokines and cell types. The improved activity of PR-957 versus etanercept in an aggressive model of CIA may also reflect pleiotropic effects; unlike TNF- $\alpha$ blockade, 
PR-957 treatment was associated with reduced autoantibody levels ${ }^{37}$. These data support the development of PR-957, a selective immunoproteasome inhibitor, as a therapeutic modality for autoimmune disorders such as rheumatoid arthritis.

\section{METHODS}

Methods and any associated references are available in the online version of the paper at http://www.nature.com/naturemedicine/.

Note: Supplementary information is available on the Nature Medicine website.

\section{ACKNOWLEDGMENTS}

We thank R. Deshaies for discussion and comments. We thank N. Shastri (University of California-Berkeley) for providing $\mathrm{T}$ cell hybridomas and V. Cerundulo (University of Oxford) for the contribution of recombinant vaccinia viruses. We acquired P14 (transgenic line 318) and RIP-GP mice from M. van den Broek, University Hospital Zurich. Psmb $8^{-1-}$ gene-targeted mice were obtained from J. Monaco, University of Cincinnati. We thank J. Mattar (Rheumatology, Ueberlingen-Nussdorf) for the collection of blood from individuals with rheumatoid arthritis and L. Klotz and P. Knolle for instructions on the $T_{H} 17$ differentiation assay. U. Beck is achnowledged for excellent technical assistance. This work was funded by the German National Science Foundation grant GR 1517/4-2 and the Swiss National Science Foundation grant 31003A_119699. K.W.K. holds a fellowship of the Graduate School Chemical Biology at University of Constance (KoRS-CB).

\section{COMPETING INTERESTS STATEMENT}

The authors declare competing financial interests: details accompany the full-text HTML version of the paper at http://www.nature.com/naturemedicine/.

1. Schwartz, A.L. \& Ciechanover, A. The ubiquitin-proteasome pathway and pathogenesis of human diseases. Annu. Rev. Med. 50, 57-74 (1999).

2. Kloetzel, P.M. Antigen processing by the proteasome. Nat. Rev. Mol. Cell Biol. 2 , 179-188 (2001).

3. Borissenko, L. \& Groll, M. 20S proteasome and its inhibitors: crystallographic knowledge for drug development. Chem. Rev. 107, 687-717 (2007).

4. Kloetzel, P.M. \& Ossendorp, F. Proteasome and peptidase function in MHC-class-Imediated antigen presentation. Curr. Opin. Immunol. 16, 76-81 (2004).

5. Griffin, T.A. et al. Immunoproteasome assembly: cooperative incorporation of interferon $\gamma($ IFN- $\gamma$ )-inducible subunits. J. Exp. Med. 187, 97-104 (1998).

6. Basler, M., Youhnovski, N., van den Broek, M., Przybylski, M. \& Groettrup, M. Immunoproteasomes down-regulate presentation of a subdominant $\mathrm{T}$ cell epitope from lymphocytic choriomeningitis virus. J. Immunol. 173, 3925-3934 (2004).

7. Basler, M., Moebius, J., Elenich, L., Groettrup, M. \& Monaco, J.J. An altered T cell repertoire in MECL-1-deficient mice. J. Immunol. 176, 6665-6672 (2006).

8. Chen, W., Norbury, C.C., Cho, Y., Yewdell, J.W. \& Bennink, J.R. Immunoproteasomes shape immunodominance hierarchies of antiviral $C D 8^{+} \mathrm{T}$ cells at the levels of $\mathrm{T}$ cell repertoire and presentation of viral antigens. J. Exp. Med. 193, 1319-1326 (2001).

9. Elliott, P.J., Zollner, T.M. \& Boehncke, W.H. Proteasome inhibition: a new antiinflammatory strategy. J. Mol. Med. 81, 235-245 (2003).

10. Richardson, P.G. et al. Bortezomib or high-dose dexamethasone for relapsed multiple myeloma. N. Engl. J. Med. 352, 2487-2498 (2005).

11. Qureshi, N. et al. The proteasome as a lipopolysaccharide-binding protein in macrophages: differential effects of proteasome inhibition on lipopolysaccharide-induced signaling events. J. Immunol. 171, 1515-1525 (2003).

12. Palombella, V.J. et al. Role of the proteasome and NF-kB in streptococcal cell wallinduced polyarthritis. Proc. Natl. Acad. Sci. USA 95, 15671-15676 (1998).
13. Berkers, C.R. et al. Activity probe for in vivo profiling of the specificity of proteasome inhibitor bortezomib. Nat. Methods 2, 357-362 (2005).

14. Chauhan, D. et al. A novel orally active proteasome inhibitor induces apoptosis in multiple myeloma cells with mechanisms distinct from bortezomib. Cancer Cell 8, 407-419 (2005).

15. Demo, S.D. et al. Antitumor activity of PR-171, a novel irreversible inhibitor of the proteasome. Cancer Res. 67, 6383-6391 (2007).

16. Bross, P.F. et al. Approval summary for bortezomib for injection in the treatment of multiple myeloma. Clin. Cancer Res. 10, 3954-3964 (2004).

17. Kuhn, D.J. et al. Potent activity of carfilzomib, a novel, irreversible inhibitor of the ubiquitin-proteasome pathway, against preclinical models of multiple myeloma. Blood 110, 3281-3290 (2007).

18. Zanelli, E., Zhou, P., Cao, H., Smart, M.K. \& David, C.S. Genomic organization and tissue expression of the mouse proteasome gene Lmp-7. Immunogenetics $\mathbf{3 8}$ 400-407 (1993).

19. Fehling, H.J. et al. MHC class I expression in mice lacking the proteasome subunit LMP-7. Science 265, 1234-1237 (1994).

20. De, M. et al. $\beta 2$ subunit propeptides influence cooperative proteasome assembly. J. Biol. Chem. 278, 6153-6159 (2003).

21. Khan, S. et al. Immunoproteasomes largely replace constitutive proteasomes during an antiviral and antibacterial immune response in the liver. J. Immunol. 167, 6859-6868 (2001).

22. Gaczynska, M., Rock, K.L., Spies, T. \& Goldberg, A.L. Peptidase activities of proteasomes are differentially regulated by the major histocompatibility complex-encoded genes for LMP2 and LMP7. Proc. Natl. Acad. Sci. USA 91, 9213-9217 (1994).

23. Ho, Y.K., Bargagna-Mohan, P., Wehenkel, M., Mohan, R. \& Kim, K.B. LMP2-specific inhibitors: chemical genetic tools for proteasome biology. Chem. Biol. 14, 419-430 (2007).

24. Palmowski, M.J. et al. Role of immunoproteasomes in cross-presentation. J. Immunol. 177, 983-990 (2006).

25. Nussbaum, A.K., Rodriguez-Carreno, M.P., Benning, N., Botten, J. \& Whitton, J.L. Immunoproteasome-deficient mice mount largely normal $\mathrm{CD}^{+} \mathrm{T}$ cell responses to Iymphocytic choriomeningitis virus infection and DNA vaccination. J. Immunol. 175 1153-1160 (2005).

26. Ohashi, P.S. et al. Ablation of 'tolerance' and induction of diabetes by virus infection in viral antigen transgenic mice. Cell 65, 305-317 (1991).

27. Pircher, H., Burki, K., Lang, R., Hengartner, H. \& Zinkernagel, R.M. Tolerance induction in double specific T-cell receptor transgenic mice varies with antigen. Nature 342, 559-561 (1989).

28. Caudill, C.M. et al. T cells lacking immunoproteasome subunits MECL-1 and LMP7 hyperproliferate in response to polyclonal mitogens. J. Immunol. 176, 4075-4082 (2006).

29. Kagari, T., Doi, H. \& Shimozato, T. The importance of IL-1 $\beta$ and TNF- $\alpha$, and the noninvolvement of IL- 6 , in the development of monoclonal antibody-induced arthritis. J. Immunol. 169, 1459-1466 (2002).

30. Van Kaer, L. et al. Altered peptidase and viral-specific T cell response in LMP2 mutant mice. Immunity 1, 533-541 (1994).

31. Klare, N., Seeger, M., Janek, K., Jungblut, P.R. \& DahImann, B. Intermediate-type 20 $S$ proteasomes in HeLa cells: 'asymmetric' subunit composition, diversity and adaptation. J. Mol. Biol. 373, 1-10 (2007).

32. Frausto, R.F., Crocker, S.J., Eam, B., Whitmire, J.K. \& Whitton, J.L. Myelin oligodendrocyte glycoprotein peptide-induced experimental allergic encephalomyelitis and T cell responses are unaffected by immunoproteasome deficiency. J. Neuroimmunol. 192, 124-133 (2007).

33. Naujokat, C. et al. Proteasomal chymotrypsin-like peptidase activity is required for essential functions of human monocyte-derived dendritic cells. Immunology 120, 120-132 (2007).

34. Qureshi, N. et al. The proteasome: a central regulator of inflammation and macrophage function. Immunol. Res. 31, 243-260 (2005).

35. Kisselev, A.F., Callard, A. \& Goldberg, A.L. Importance of the different proteolytic sites of the proteasome and the efficacy of inhibitors varies with the protein substrate. J. Biol. Chem. 281, 8582-8590 (2006).

36. Strehl, B. et al. Immunoproteasomes are essential for clearance of Listeria monocytogenes in nonlymphoid tissues but not for induction of bacteria-specific CD8 ${ }^{+}$ T cells. J. Immunol. 177, 6238-6244 (2006).

37. Williams, R.O., Marinova-Mutafchieva, L., Feldmann, M. \& Maini, R.N. Evaluation of TNF- $\alpha$ and IL-1 blockade in collagen-induced arthritis and comparison with combined anti-TNF- $\alpha$ /anti-CD4 therapy. J. Immunol. 165, 7240-7245 (2000). 


\section{ONLINE METHODS}

Reagents. We discovered PR-957 as part of a medicinal chemistry effort to identify tripeptide ketoepoxide proteasome inhibitors with selectivity for LMP7. We determined selectivity ratios of new compounds in one or more of the following assays: inhibition of purified human constitutive proteasomes and immunoproteasomes (described below), inhibition of chymotrypsin-like activity in lysates derived from tumor cells expressing predominantly constitutive proteasome or immunoproteasome, and proteasome active-site ELISA (Supplementary Methods). We synthesized PR-957, PR-825 and the biotinylated active-site probe PR-584 as previously described ${ }^{38}$. We synthesized carfilzomib as previously described ${ }^{15}$. We purchased purified human $20 \mathrm{~S}$ proteasomes and immunoproteasomes from Boston Biochem. We purchased etanercept from a local pharmacy.

Mice and viruses. We purchased $\mathrm{C} 57 \mathrm{BL} / 6$ mice $\left(\mathrm{H}-2^{\mathrm{b}}\right)$ and $\mathrm{BALB} / \mathrm{c}$ mice $\left(\mathrm{H}-2^{\mathrm{d}}\right)$ from Charles River Laboratories. We acquired P14 (transgenic line 318$)^{27}$ and RIP-GP mice ${ }^{26}$ from M. van den Broek. We acquired $P s m b 8^{-1-}$ gene-targeted mice ${ }^{19}$ from J. Monaco. We purchased DBA1/J mice from Taconic. We performed all experiments under protocols approved by The Animal Experimentation Review Board of Regierungspräsidium Freiburg and the Proteolix Institutional Animal Care and Use Committee. We propagated rVV-UTY (kindly provided by V. Cerundulo) on BSC40 cells. We originally obtained LCMV-WE from F. Lehmann-Grube and propagated it on the fibroblast line L929 (from M. van den Broek). We infected mice with 200 PFU LCMV-WE i.v. or $2 \times 10^{6}$ PFU rVV-UTY intraperitoneally.

Cells. We obtained MOLT-4 (human acute lymphocytic leukemia) and A20 (mouse lymphoma) cells from American Type Culture Collection and cultured them in medium recommended by the supplier. We purchased human PBMCs from normal healthy volunteers from AllCells, and we obtained PBMCs from individuals with rheumatoid arthritis (who had given informed consent) from a rheumatologist (J. Mattar) after approval by the Institutional Review Board of the University of Constance.

Fluorogenic 20S proteasome assays. We purified and analyzed $20 \mathrm{~S}$ proteasomes from livers of LCMV-infected mice ( $8 \mathrm{~d}$ after infection with 200 PFU of LCMV-WE i.v.) as described previously ${ }^{39}$. We performed hydrolytic assays for proteasome activity of mouse proteasomes and purified human constitutive proteasome and immunoproteasome as previously described ${ }^{15,39}$

Peripheral blood mononuclear cell and splenocyte stimulation. After compound exposure, we stimulated PBMCs and mouse splenocytes with LPS (Escherichia coli, O111:B4, Sigma) at $1 \mu \mathrm{g} \mathrm{ml}^{-1}$ and $5 \mu \mathrm{g} \mathrm{ml}^{-1}$, respectively, for $24 \mathrm{~h}$, and analyzed supernatants for IL-23 (eBiosciences), TNF- $\alpha$ (BD Bioscience) and IL-6 (BD Bioscience) by ELISA. We analyzed PBMCs and splenocytes stimulated with antibodies to CD3 and CD28 for $24 \mathrm{~h}$ for production of IFN- $\gamma$ and IL-2 (PBMC) or IFN- $\gamma$ (splenocytes) by ELISA (BD Bioscience). We measured expression of CD25 on the CD4 subset in PBMC by flow cytometry (BD Pharmingen).

T helper type 17 cell differentiation. We prepared $\mathrm{CD}^{+} \mathrm{T}$ cells from splenocytes by positive selection via magnetic cell sorting (MACS; Miltenyi Biotech). We stimulated cells $\left(7.5 \times 10^{4}\right.$ per well $)$ with antibodies to $\mathrm{CD} 3$ and CD28 in the presence of $2.5 \mathrm{ng} \mathrm{ml}^{-1}$ TGF- $\beta, 30 \mathrm{ng} \mathrm{ml}^{-1}$ IL-6 (eBioscience) and antibodies to IL-4 and IFN- $\gamma$ (eBioscience) for 3 days. We measured intracellular IL-17A expression after $4 \mathrm{~h}$ exposure to $5 \mathrm{ng} \mathrm{ml}^{-1}$ phorbol 12-myristate 13-acetate and $200 \mathrm{ng} \mathrm{ml}^{-1}$ ionomycin in the presence of brefeldin A (Sigma).

Antigen-specific T cell assays. We cultured $1 \times 10^{5} \mathrm{UTY}_{246-254}-$ specific LacZexpressing $\mathrm{T}$ cell hybridoma cells (kindly contributed by N. Shastri) overnight with 3-10 × $10^{5}$ stimulator cells in 96-well plates overnight and analyzed them as detailed previously ${ }^{7}$. We generated LCMV-specific CTL lines exactly as previously described ${ }^{40}$

Proteasome inhibition in mice. We formulated PR-957 in an aqueous solution of $10 \%(\mathrm{wt} / \mathrm{vol})$ sulfobutylether- $\beta$-cyclodextrin and $10 \mathrm{mM}$ sodium citrate ( $\mathrm{pH} 3.5)$ and administered it to mice as a single i.v. bolus. We collected whole blood (sodium heparin anti-coagulant) and tissue samples (kidney, heart and spleen) $1 \mathrm{~h}$ after administration and processed them as previously described ${ }^{15}$ for protein quantification and proteasome activity determination by activesite ELISA.

Treatment of RIP-GP mice. We purified Thy $1.2^{+}$cells from splenocytes of TCR transgenic P14 mice ${ }^{27}$ according to the manufacturer's protocol (Milteny Biotec). We injected Thy $1.2^{+}$cells $\left(7.5 \times 10^{6}\right)$ purified from P14 mice i.v. into RIP-GP mice (day 0). On day 1, we infected the mice with 200 PFU LCMV-WE i.v. and treated them with PR-957 at $6 \mathrm{mg}$ per $\mathrm{kg}$ body weight for $10 \mathrm{~d}$ (days 0-4 i.v. and days 5-9 intraperitoneally). We measured glucose in the blood on days $1,3,6,7,8$ and 9 after infection using the Glycaemie C Medi-Test (Macherey-Nagel).

Arthritis models. We induced CIA in 8- to 11-week-old male DBA1/J mice (Taconic Farms) by an intradermal injection at the base of the tail with $100 \mu \mathrm{g}$ of denatured type II bovine collagen (Chondrex) emulsified in complete Freund's adjuvant containing $4 \mathrm{mg} \mathrm{ml}^{-1}$ heat-denatured mycobacterium (Chondrex). We induced CAIA in 5- to 6-week-old female BALB/c mice (kept on breeder chow) by an i.v. administration of $1 \mathrm{mg}$ of a cocktail of four antibodies against type II collagen (Chemicon) followed by intraperitoneal challenge with $50 \mu \mathrm{g}$ LPS on day 3 . We initiated treatment after we observed clinical signs of arthritis (day 25 for the CIA model and day 4 for the CAIA model). We scored paws for disease severity on a 0 (no disease) to 4 (maximal swelling) scoring system and summed the paw scores to yield individual mouse scores.

Statistical analyses. For comparisons of treatment groups, we performed the unpaired $t$ test (Mann-Whitney), paired $t$ tests and one-way or two-way ANOVA (where appropriate). For ANOVA, we used Bonferroni post hoc analysis to compare treatment groups. We performed all statistical analyses with GraphPad Prism Software (version 4.01). Statistical significance was achieved when $P$ was less than 0.05 .

38. Shenk, K.D. et al. Compounds For Enzyme Inhibition. US Patent 2007/0293465 A1, (2008).

39. Groettrup, M. et al. The interferon- $\gamma$-inducible $11 \mathrm{~S}$ regulator (PA28) and the LMP2 LMP7 subunits govern the peptide production by the $20 \mathrm{~S}$ proteasome in vitro. J. Biol. Chem 270, 23808-23815 (1995).

40. Basler, M. \& Groettrup, M. No essential role for tripeptidyl peptidase II for the processing of LCMV-derived T cell epitopes. Eur. J. Immunol. 37, 896-904 (2007) 